\title{
Isotermas generadas en soldadura de aluminio 6061-T6 con enfriamiento acelerado
}

\author{
Isotherms Generated in 6061-T6 Aluminum Weld with Accelerated Cooling \\ César Mendoza-Gómora ${ }^{a}$, Ricardo R. Ambriz-Rojas ${ }^{b}$
}

\begin{abstract}
:
The isotherms generated by the welding process in 6061-T6 aluminum alloy were obtained by means of finite element using the ANSYS program (APDL platform). In addition, a mathematical model (thin plate) that governs the heat source motion behavior was applied. The analytical and numerical simulation results showed that the maximum width of the isotherm at which the greatest overaging occurred was approximately $10 \mathrm{~mm}$ from the center of the weld bead, however, the analytical results did not show an acceptable correlation with the simulated results.
\end{abstract}

Keywords:

Simulation, 6061-T6 aluminum, welding, isotherms, over-aging

\section{Resumen:}

Por medio del estudio por elemento finito se obtuvieron las isotermas generadas por el proceso de soldadura en la aleación de aluminio 6061-T6 empleando el programa ANSYS (plataforma APDL). Aunado a este estudio se aplicó un modelo matemático (para placa delgada) que rige el comportamiento de una fuente de calor en movimiento. Los resultados analíticos y por simulación numérica mostraron que el ancho máximo de la isoterma al cual ocurre el mayor sobre envejecimiento fue aproximadamente $10 \mathrm{~mm}$ a partir del centro del cordón de soldadura, sin embargo, de manera general los resultados analíticos no presentaron una correlación aceptable con los simulados.

Palabras Clave:

Simulación, aluminio 6061-T6, soldadura, isotermas, sobreenvejecimiento

\section{Introducción}

La aleación Al 6061-T6 (Al-Mg-Si) se puede tratar térmicamente por solubilización y envejecimiento artificial o natural para obtener una alta resistencia a la tensión respecto al aluminio sin elementos de aleación. Las aplicaciones más importantes de este material se encuentran en la industria aeronáutica y automotriz, en la fabricación de trenes de alta velocidad y estructuras 1,2,3. También se usa para la fabricación de líneas de tuberías y recipientes para productos químicos debido a su buena resistencia a la corrosión.

La resistencia a la tensión de esta aleación (310 MPa) puede incluso superar a la de un acero estructural A 36 . Esta característica es obtenida a partir de un tratamiento térmico de precipitación, en donde primeramente se lleva a la aleación por encima de la línea de solvus $\left(530^{\circ} \mathrm{C}\right)$ para disolver los elementos aleantes. Posteriormente, se realiza un enfriamiento súbito para retener a los átomos de soluto en la matriz de aluminio, con lo que se tiene una solución sólida sobresaturada. Finalmente, se presenta la difusión de los elementos de aleación (Mg y Si) para que se lleve a cabo la nucleación y crecimiento de precipitados $\beta^{\prime \prime}$ finos, duros y dispersos dentro de la matriz de aluminio (fenómeno de envejecimiento) que deforman a la red cristalina proporcionando el máximo grado de endurecimiento.

Debido a que esta aleación es tratable térmicamente, su uso es limitado en aplicaciones a temperaturas por encima de los $200^{\circ} \mathrm{C}$, debido a que los precipitados $\beta^{\prime \prime}$ crecen y cambian de morfología formando precipitados $\beta^{\prime}$ (fenómeno de sobreenvejecimiento), perdiendo la distorsión generada durante el envejecimiento, lo cual se refleja en la disminución de sus propiedades mecánicas.

Autor de Correspondencia, Universidad Autónoma del Estado de Hidalgo, Escuela Superior Ciudad Sahagún, Email: cesar_mendoza@uaeh.edu.mx

b Instituto Politécnico Nacional, Centro de Investigación e Innovación Tecnológica, Email: rrambriz@ipn.mx 
Por otro lado, es indispensable la unión de esta aleación. Los procesos de soldadura por arco eléctrico son los más usados, estos aportan una cantidad de energía necesaria para fundir el material de aporte y el metal base, sin embargo, este calor repercute en las condiciones microestructurales de la unión soldada que se ven reflejados en el cambio de propiedades mecánicas en la zona afectada térmicamente (ZAT).

La problemática generada en las aleaciones de aluminio por los procesos de soldadura se ha estudiado ampliamente, desde lo referente al cordón de soldadura como agrietamiento en caliente, refinamiento de grano, agitación electromagnética, etc. así como la ZAT en cuanto a disminución de propiedades mecánicas a causa del sobreenvejecimiento. Sin embargo, no existen reportes sobre las isotermas generadas durante el proceso de soldadura, las cuales dan un parámetro de las zonas donde se localiza el mayor decremento en dureza (sobreenvejecimiento) en función de las temperaturas que se alcanzan 4,5,6.

En el presente proyecto de investigación se analiza la distribución de temperaturas en la soldadura de placas de Al 6061-T6 de $6.4 \mathrm{~mm}$ de espesor, mediante simulación numérica y de manera analítica. Durante el estudio del proceso de soldadura se empleó un bloque de respaldo disipador con la finalidad de generar enfriamiento acelerado en las placas soldadas y así disminuir el sobreenvejecimiento.

\section{Material y métodos}

Placas de aluminio 6061-T6 de $6.4 \mathrm{~mm}$ de espesor fueron unidas por el proceso de soldadura GMAW, donde los parámetros operativos fueron reportados anteriormente 7 . El registro de la corriente consumida durante el proceso de soldadura se realizó mediante un sensor de efecto Hall colocado en la salida del polo negativo del equipo de soldar. Este sensor midió el voltaje generado por el paso de la corriente por el conductor. Posteriormente, la digitalización de la corriente se realizó por medio de una tarjeta de adquisición de datos NI 9219 de National Instruments con una frecuencia de $75 \mathrm{~Hz}$, la cual se conectó a un puerto USB de una computadora, donde se registraron datos con el software $\mathrm{LAB}-\mathrm{VIEW}$ para su procesamiento. Un bloque de respaldo disipador fue utilizado para generar el enfriamiento acelerado durante el proceso de soldadura.

Para extraer calor se construyó un bloque disipador de aluminio. Se realizó una distribución triangular de las perforaciones internas (Figura 1) donde el flujo del fluido fue de un paso.

Sobre los cabezales se instalaron boquillas de entrada y salida del refrigerante comercial etilenglicol a una concentración del $50 \%$ que fluyó a $21 \mathrm{~mL} \mathrm{~s}^{-1}$. La Tabla 1 muestra las propiedades termofísicas del refrigerante a distintas temperaturas. Sobre estos puntos se colocaron termopares para obtener el registro de temperatura del fluido durante el proceso de soldadura. Adicionalmente se colocaron dos termopares sobre los costados del bloque para medir la temperatura inicial del bloque para considerarla en la simulación numérica. Para el registro de temperaturas, se utilizó una tarjeta de adquisición de datos NI 9213 de National Instruments, la cual acondiciona la señal de voltaje y establece una medición de la temperatura directa. Esta tarjeta se conectó a una computadora y la digitalización de la señal se llevó a cabo por medio de un programa en LabVIEW a $75 \mathrm{~Hz}$.
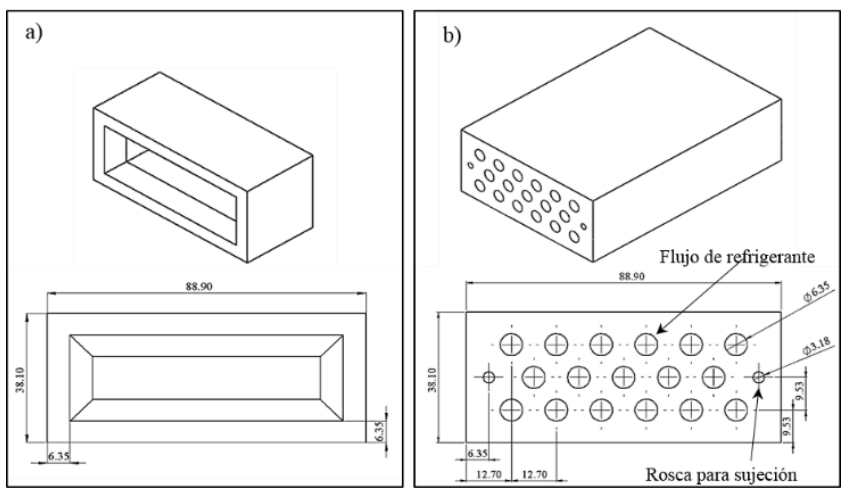

Figura 1. Dimensiones del bloque disipador, a) cabezales y b) coraza. Acotaciones en $\mathrm{mm}$.

\begin{tabular}{lllc}
\hline $\begin{array}{l}\text { Temperatura } \\
\left({ }^{\circ} \mathbf{C}\right)\end{array}$ & $\begin{array}{l}\text { Viscosidad dinámica, } \mu \\
\left(\mathbf{k g ~ m}^{-1} \mathbf{~ s}^{-1}\right)\end{array}$ & $\begin{array}{l}\text { Capacidad calorífica, } \boldsymbol{C}_{\boldsymbol{p}} \\
\left(\mathbf{J ~ k g}^{-1}{ }^{\circ} \mathbf{C}^{-1}\right)\end{array}$ & $\begin{array}{c}\text { Densidad, } \boldsymbol{\rho} \\
\left(\mathbf{k g ~ m}^{-\mathbf{3}}\right)\end{array}$ \\
\hline-17.8 & 0.0220 & 3265 & 1100 \\
-11 & 0.0176 & 3283 & 1096 \\
4.40 & 0.0065 & 3328 & 1088 \\
26.7 & 0.0028 & 3412 & 1077 \\
\hline
\end{tabular}

Tabla 1. Propiedades termofísicas del etilglicol al $50 \%$ en función de la temperatura (8).

Con la finalidad de predecir las isotermas generadas durante el proceso de soldadura se llevó a cabo el análisis por medio de elemento finito mediante el software ANSYS, donde fueron empleados los resultados obtenidos durante la experimentación (temperatura inicial del bloque y corriente). El tipo de análisis a solucionar es térmicotransiente en tres dimensiones, por lo que se seleccionó un elemento sólido (SOLID 278), el cual sirve para realizar análisis en 3-D con un solo grado de libertad (temperatura) y puede utilizarse para solucionar problemas en estado estable o transiente. La Tabla 2 indica las propiedades termofísicas del Al 6061-T6 para realizar los cálculos en 
función de la temperatura. El calor de aporte real suministrado fue de $3397 \mathrm{~W}$. Entre los diferentes modelos de distribución de calor, semiesférica, gaussiana, elipsoidal y de doble elipse se eligió una distribución de calor gaussiana (Ecuación 1), donde $q$ es el flux de calor $\left(\mathrm{W} \mathrm{\textrm {m } ^ { - 2 }}\right), \mathrm{Q}$ es el calor de aporte $(\mathrm{W})$, a es el radio efectivo de la fuente de calor $(\mathrm{m})$ y $R^{*}$ es la distancia radial al punto donde se requiere calcular el flux de calor $(\mathrm{m})$. Se utilizó un coeficiente de convección constante de $40 \mathrm{~W} \mathrm{~m}^{-2}{ }^{\circ} \mathrm{C}^{-1}$. Se consideró la temperatura inicial de las placas de $5^{\circ} \mathrm{C}$, que fue la que registraron los termopares antes de comenzar el proceso de soldadura 9,10,11,12.

\begin{tabular}{lllc}
\hline $\begin{array}{l}\text { Temperatura } \\
\left({ }^{\circ} \mathbf{C}\right)\end{array}$ & $\begin{array}{l}\text { Conductividad térmica, } \kappa \\
\left(\mathbf{W ~ m ~ m}^{-1}{ }^{\circ} \mathbf{C}^{-1}\right)\end{array}$ & $\begin{array}{l}\text { Capacidad calorífica, } c p \\
\left(\mathrm{~J} \mathrm{~kg}^{-1}{ }^{\circ} \mathrm{C}^{-1}\right)\end{array}$ & $\begin{array}{c}\text { Densidad, } \rho \\
\left(\mathrm{kg} \mathrm{m}^{-3}\right)\end{array}$ \\
\hline 25 & 167 & 870 & 2705 \\
100 & 195 & 950 & 2695 \\
200 & 203 & 980 & 2675 \\
300 & 211 & 1020 & 2665 \\
400 & 212 & 1060 & 2635 \\
500 & 225 & 1150 & 2610 \\
600 & 200 & 1160 & 2590 \\
\hline
\end{tabular}

Tabla 2. Propiedades termofísicas del Al 6061-T6 en función de la temperatura (13).

$$
q=\frac{3 Q}{\pi a^{2}} \exp \left[\frac{R^{* 2}}{a^{2} / 3}\right]
$$

El modelo geométrico de la junta soldada se discretizó por medio de elementos hexaédricos. En la zona de fusión se refinó la malla para tener una distribución de temperaturas más precisa con respecto al resto del modelo. La Figura 2 muestra la discretización de elementos finitos del modelo geométrico. La temperatura inicial del bloque se estableció en base a las temperaturas registradas por los termopares situados en los costados del mismo durante la experimentación.

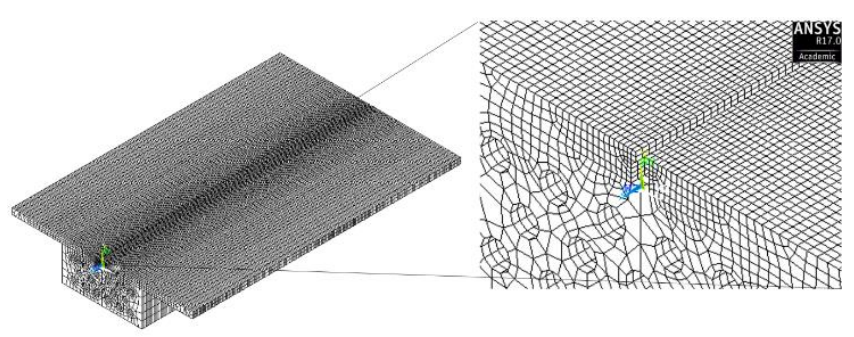

Figura 2. Mallado para el análisis térmico-transiente-no lineal de la fuente de calor sobre aluminio 6061-T6 ER.
Para determinar el tamaño y morfología de las isotermas analíticas se recurrió a parámetros adimensionales ya que simplifican el estudio analítico, los cuales pueden ser graficados como se ilustra en la Figura 3, donde $n$ es el parámetro adimensional de operación y $\delta$ de espesor, $\xi$ y $\psi$ son las coordenadas adimensionales en $X$ y $Y$ respectivamente y $\sigma_{m}$ es el vector adimensional de radio. Un resumen del uso de los parámetros adimensionales se muestra en un estudio previo, donde se obtienen las isotermas analíticas del proceso de soldadura sin enfriamiento acelerado 14,7 .

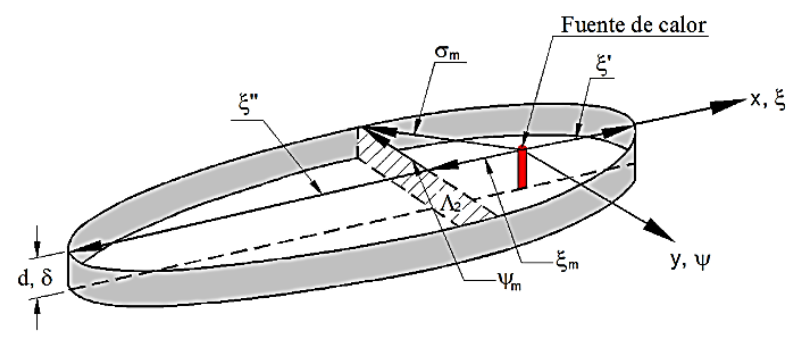

Figura 3. Representación gráfica de las dimensiones de una sección isotérmica para una placa delgada, de acuerdo con parámetros adimensionales derivados de la solución de Rosenthal 14.

A partir de la instrumentación colocada en el bloque de respaldo disipador se pudo determinar la cantidad de calor extraída en un punto y tiempo en específico, estos resultados fueron útiles para determinar un espesor de placa equivalente, es decir, un espesor de material capaz de disipar el calor que extrajo el bloque de respaldo disipador y con este espesor más el de la placa se aplicó el modelo de placa delgada haciendo uso de los números adimensionales. El modelo general para la distribución de temperatura en una placa delgada consiste de un cuerpo isotrópico semi-infinito con una temperatura inicial $T_{0}$, y está limitado a una dirección en un plano. La fuente de calor funde el espesor del cuerpo y por consiguiente el calor se disipa sólo en dos direcciones. Las propiedades termofísicas son constantes y no dependen de la temperatura. El calor perdido por radiación y convección son consideradas en la eficiencia del propio proceso de soldadura. A partir de la Ecuación 2 es posible obtener el espesor de placa equivalente. Originalmente esta ecuación se aplica al modelo de placa intermedia, donde por medio de esta ecuación es posible determinar el espesor de placa crítico, es decir, el espesor que indica el límite entre el modelo de placa delgada y el modelo de placa gruesa. Durante la investigación los autores utilizaron esta ecuación de manera inversa para determinar un calor de entrada para una placa de espesor 
dado. Ante este aspecto, se analizó la posibilidad de emplear esta ecuación para determinar el espesor de placa equivalente para este proyecto 15.

$$
d_{c}=\left[\frac{Q / v}{2 \rho C_{p}}\left(\frac{1}{T_{1}-T_{0}}+\frac{1}{T_{2}-T_{0}}\right)\right]^{1 / 2}
$$

donde:

$d_{c}=$ Espesor crítico de la placa, $\mathrm{m}$

$Q=$ Calor de aporte, $W$

$v=$ Velocidad de avance, $\mathrm{m} \mathrm{s}^{-1}$

$\rho=$ Densidad, $\mathrm{kg} \mathrm{m}^{-3}$

$C_{p}=$ Capacidad calorífica, $\mathrm{J} \mathrm{kg}^{-1}{ }^{\circ} \mathrm{C}^{-1}$

$T_{o}=$ Temperatura inicial, ${ }^{\circ} \mathrm{C}$

Las temperaturas de $T_{1}$ y $T_{2}$ son las temperaturas entre las cuales ocurren cambios microestructurales significativos. Entonces, considerando que se tenía un aluminio $6061-\mathrm{T} 6$, estas temperaturas fueron 380 y $630^{\circ} \mathrm{C}$ respectivamente, rango en el cual se suscitan los cambios más notorios en la aleación en estudio.

\section{Análisis y discusión de resultados}

En un proceso de soldadura por arco eléctrico, el calor de aporte depende de la velocidad de avance, voltaje, eficiencia del proceso y de la corriente suministrada. Durante el proceso de soldadura GMAW la corriente depende principalmente de la alimentación constante del electrodo mediante un sistema mecánico, por lo tanto pueden existir fluctuaciones en las mediciones de corriente que pueden afectar durante el proceso de soldadura. Lo anterior conllevó a la adquisición de datos de corriente en tiempo real en la soldadura de placas de aluminio 6061-T6. La Figura 4 muestra el comportamiento de la corriente en función de la distancia durante la soldadura.

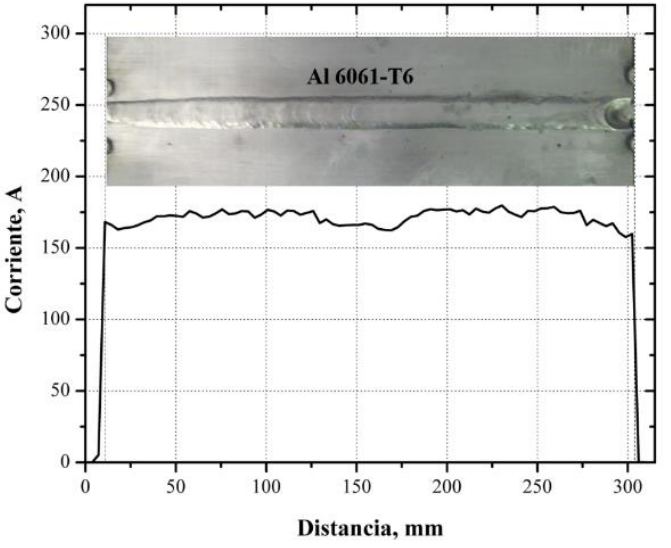

Figura 4. Señal de corriente del arco eléctrico durante el proceso de soldadura.

El valor promedio fue de 171 A con una desviación estándar de 5 A (2.85\%). Esta ligera variación de la corriente no mostró cambios significativos en la morfología del cordón de soldadura, como se puede ver en la Figura 4 que muestra un cordón de soldadura sano, lo que indica también que no hay gran variación en el calor de aporte (3397 W).

Durante el proceso de soldadura con enfriamiento acelerado se colocó un termopar a la salida del bloque de respaldo disipador el cual registró el diferencial de temperaturas del refrigerante (ver Figura 5), que aunado al flujo másico utilizado, fue posible determinar la cantidad de calor extraída mediante la Ecuación 3. Las propiedades termofísicas del refrigerante (Tabla 1 ) se consideraron constantes a $-2.5^{\circ} \mathrm{C} 16$.

$$
Q_{E}=\dot{m} C p \Delta T
$$

donde:

$\dot{m}=$ Flujo másico, $\mathrm{kg} \mathrm{s}^{-1}$

$Q_{E}=$ Calor extraído, W

$\Delta T=$ Diferencial de temperatura, ${ }^{\circ} \mathrm{C}$

La cantidad de calor extraída por el refrigerante fue de 489 $\mathrm{J} \mathrm{s}^{-1}$, ésta fue sustituida por el calor de aporte en la Ecuación 2 y así se determinó un espesor de placa equivalente de $3.9 \mathrm{~mm}$ que sumado con el espesor inicial de $6.4 \mathrm{~mm}$ se obtuvo finalmente un espesor de $10.3 \mathrm{~mm}$. Este valor se consideró en los números adimensionales para obtener las isotermas analíticas. 


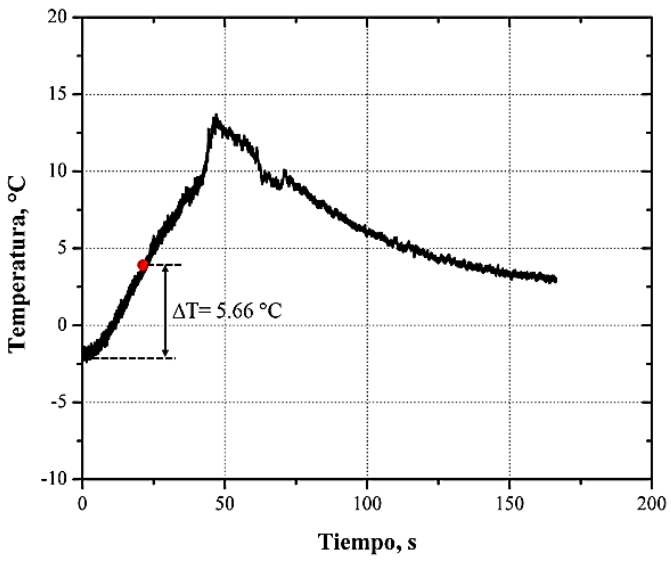

Figura 5. Evolución de la temperatura en función del tiempo del refrigerante durante el proceso de soldadura.

Una vista isométrica de las isotermas obtenidas por simulación numérica se presenta en la Figura 6, de igual manera se muestra un corte en dirección longitudinal al avance de la fuente de calor, donde se logra observar la distribución de temperaturas tanto en la placa como en el bloque de respaldo disipador.

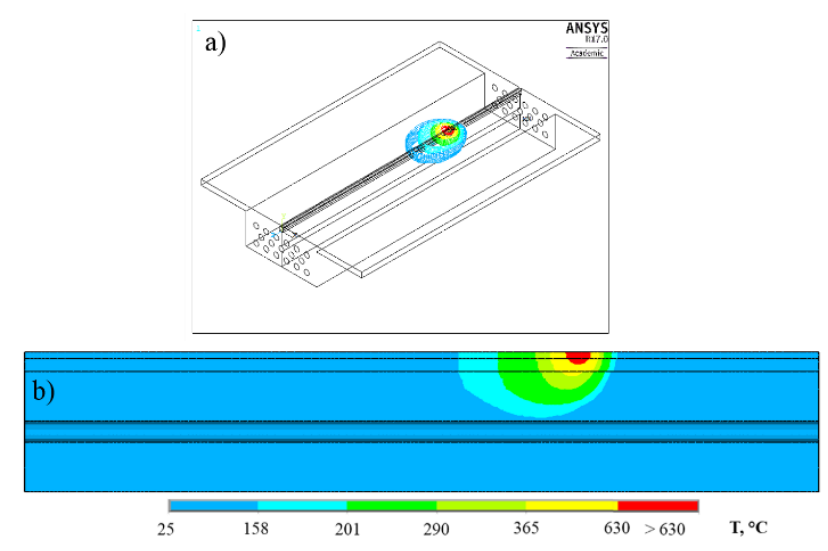

Figura 6. Isotermas obtenidas por simulación numérica a) vista isométrica y b) vista lateral.

Una correlación entre la morfología y tamaño de las isotermas obtenidas por simulación numérica y de manera analítica fue realizada, como se puede ver en la Figura 7. En el estudio previo se pudo constatar que la isoterma crítica a la que ocurre el mayor sobreenvejecimiento es la de aproximadamente $380^{\circ} \mathrm{C}$ y el ancho máximo de esta isoterma sin enfriamiento acelerado fue aproximadamente $18 \mathrm{~mm}$ a partir del centro del cordón de soldadura. Como se puede observar, con el uso del enfriamiento acelerado se disminuye el tamaño de la ZAT debido a que el ancho máximo de la isoterma $\left(365-380^{\circ} \mathrm{C}\right)$ a la que se lleva a cabo el sobreenvejecimiento reduce de $18 \mathrm{~mm}$ a $10 \mathrm{~mm}$, sin embargo, se puede constatar de manera indirecta con la temperatura de $365^{\circ} \mathrm{C}$ que sigue existiendo el mismo grado de sobreenvejecimiento dentro de la ZAT. En otras palabras, con el enfriamiento acelerado lo que se genera es que la temperatura a la cual se lleva a cabo el mayor sobreenvejecimiento (mayor pérdida de dureza) se desplace hacia el centro del cordón de soldadura, pero eso no evita que deje de existir el sobreenvejecimiento 7 . Las isotermas analíticas fueron obtenidas a partir de los números adimensionales y considerando el espesor de placa equivalente. Como puede notarse la morfología es aceptable, sin embargo, el tamaño de las isotermas presentan un porcentaje de error considerable (ver Tabla 3) que indica que el modelo matemático no es el adecuado para este tipo de análisis que emplea un enfriamiento acelerado.



Figura 7. Correlación de las isotermas obtenidas de manera analítica y por simulación numérica con enfriamiento acelerado. Acotación en $\mathrm{mm}$.

\begin{tabular}{ll}
\hline Isoterma, ${ }^{\circ} \mathrm{C}$ & ${ }^{\%} E_{A}^{S}$ \\
\hline 630 & 85 \\
365 & 29.89 \\
290 & 0.5 \\
201 & 61 \\
158 & 108 \\
\hline
\end{tabular}

Tabla 3. Porcentajes de error de las isotermas analíticas respecto de las isotermas simuladas con enfriamiento rápido. 
Como se puede ver, el uso de los números adimensionales para la determinación de las isotermas no ha tenido una buena aceptación para este estudio, esto se debe principalmente a la consideración del uso de placas semi-infinitas, y que en conjunto con el alto coeficiente de conductividad térmica del aluminio (167 W m $\mathrm{m}^{-1}{ }^{\circ} \mathrm{C}^{-1}$ ) generan un enfriamiento muy rápido, generando que el tamaño de las isotermas de baja temperatura sean cada vez más grandes. Cabe señalar que este modelo matemático no aplica a la zona de fusión, que es válido a distancias cercanas al cordón de soldadura y que aplica sobre una placa plana. Ante este aspecto, se abre un campo de estudio en el área del modelo matemático de fuentes de calor en movimiento, al considerar soldaduras de aluminio con diseño de junta.

\section{Conclusiones}

La instrumentación es una herramienta útil que permitió obtener información en tiempo real para poder realizar la simulación numérica del proceso de soldadura.

La simulación numérica permitió determinar la morfología y tamaño de las isotermas generadas durante el proceso de soldadura.

El enfriamiento acelerado durante el proceso de soldadura en aluminio 6061-T6 disminuye el ancho de la ZAT a 10 $\mathrm{mm}$ a partir del centro del cordón de soldadura, sin embargo, no disminuye el grado de sobreenvejecimiento ya que la isoterma crítica sigue existiendo, sólo que a una distancia más cercana al centro de la soldadura.

El modelo de placa delgada para obtener las isotermas analíticas mediante los parámetros adimensionales no se ajusta a soldaduras de aluminio con diseño de junta y enfriamiento acelerado.

\section{Agradecimientos}

Los autores agradecen al consejo nacional de ciencia y tecnología (CONACYT) por el apoyo económico otorgado para poder llevar a cabo este proyecto de investigación.

\section{Referencias}

[1] Benedyk JC. International temper designation systems for wrought aluminum alloy. Light Metal Age 2010:16-22.

[2] Heinz A, Haszler A, Keidel C, Moldenhauer S, Benedictus R, Miller WS. Recent develoment in aluminum alloys for aerospace applications. Materials Science and Engineering 2000;A A280:102-7.

[3] World Aluminium. [Consultado el 25, mayo, 2018]. Disponible en http://www.world-aluminium.org/.

[4] Kou S, Le Y. Nucleation mechanism and grain refining of weld metal. Welding Journal 1986;12:305-13.
[5] Li Y, Murr LE, Clure JCM. Flow visualization and residual microstructures associated with the friction-stir welding of 2024 aluminum to 6061 aluminum. Materials Science and Engineering 1999;A 271:213-23.

[6] Malin V. Study of metallurgical phenomena in the HAZ of 6061-T6 aluminium welded joints. Welding Journal 1995;74(9):305-18.

[7] Gómora CM, Ambriz RR, Curiel FF, Jaramillo D. Heat distribution in welds of a 6061-T6 aluminum alloy obtained by modified indirect electric arc. Journal of materials processing technology 2017;243:43341.

[8] Engineeringtoolbox. [Consultado el 12, enero, 2017]. Disponible en www.engineeringtoolbox.com/ethylene-glycol-d_146.html.

[9] Goldak J, Chakravarti A, Bibby M. A new finite element model for welding heat sources. Metallurgical Transactions B 1984;15B:299-305.

[10] Kou S. Welding Metallurgy. John Wiley \& Sons.Second ed.USA; 2003: 57-8.

[11] Soundararajan V, Zekovic S, Kovacevic R. Thermo-mechanical model withadaptive boundary conditions for friction stir welding of $\mathrm{Al} 6061$. International Journal of Machine Tools \& Manufacture 2005;45:157787.

[12] Moraitis GA, Labeas GN. Residual streess and distortion calculation of laser beam welding for aluminum lap joints. Journal of Materials Processing Technology 2008;198:260-9.

[13] Mills KC. Recommended values of thermophysical properties for selected commercial alloys. ASM International 2002.

[14] Grong O. Metallurgical modelling of welding. Materials modelling series. Second ed. Norway: 1994.

[15] Poorhaydari K, Patchett BM, Ivey DG. Estimation of cooling rate in the welding of plates with intermediate thickness. The welding journal 2005:149-55.

[16] Incropera FP, Dewitt DP. Introduction to heat transfer. second ed. N.Y.; USA: John Wiley and Sons; 1990. 